\title{
Analysis of risk factors and model establishment of recurrence after endometrial polypectomy
}

\author{
Jinglan Liu ${ }^{1,2 \#}$, Yan Liang ${ }^{1,2 \#}$, Jing Ouyang ${ }^{1,2}$, Siqin Yang ${ }^{1,2}$ \\ ${ }^{1}$ Department of Obstetrics and Gynecology, The International Peace Maternity and Child Health Hospital, School of Medicine, Shanghai Jiao Tong \\ University, Shanghai, China; ${ }^{2}$ Shanghai Key Laboratory of Embryo Original Diseases, Shanghai, China \\ Contributions: (I) Conception and design: S Yang, J Liu; (II) Administrative support: S Yang; (III) Provision of study materials or patients: Y Liang; (IV) \\ Collection and assembly of data: All authors; (V) Data analysis and interpretation: J Liu, J Ouyang; (VI) Manuscript writing: All authors; (VII) Final \\ approval of manuscript: All authors. \\ "These authors contributed equally to this work. \\ Correspondence to: Siqin Yang. Department of Obstetrics and Gynecology, The International Peace Maternity and Child Health Hospital, School of \\ Medicine, Shanghai Jiao Tong University, Hengshan Road 910, Shanghai 200030, China. Email: ysqmm123456@163.com.
}

Backgrounda Currently, determining the postoperative recurrence of endometrial polyps is an unresolved issue, so we screened the risk factors to establish a nomogram for clinical practice.

Methods: From January 2012 to June 2020, 133 patients who underwent hysteroscopic endometrial polypectomy and diagnostic curettage due to suspicious endometrial polyps for the first time and were pathologically diagnosed as endometrial polyps after surgery. All patients were followed up for more than 12 months, and $9(6.77 \%)$ cases were lost to follow-up. Logistic regression analysis of clinical factors was performed to screen independent risk factors and construct a column chart to predict the probability of adverse events. The fitting curve was used to validate the line graph.

Results: The ratio of patients with body mass index $(\mathrm{BMI})<28 \mathrm{~kg} / \mathrm{m}^{2}$ in the recurrence group was lower than in the non-recurrence group $(\mathrm{P}=0.028)$, as was the proportion of patients with polyp $<2 \mathrm{~cm}$ (recurrence group vs. non-recurrence group, $\mathrm{P}=0.027$ ) and the proportion of patients in the recurrence group treated with progesterone after surgery compared with non-recurrence group $(\mathrm{P}=0.003)$. However, the proportion of endometrial thickening in the recurrence group was higher than in the non-recurrence group $(\mathrm{P}=0.006)$. Age, $\mathrm{BMI}$, polyp size, and endometrial thickening were independent risk factors for recurrence after endometrial polypectomy [odds ratio $(\mathrm{OR})>1, \mathrm{P}<0.05$ ]. Progesterone therapy after surgery was an independent protective factor for recurrence after endometrial polypectomy $(\mathrm{OR}<1, \mathrm{P}<0.05)$. Our nomogram was based on age, $\mathrm{BMI}$, polyp size, endometrial thickening, and postoperative progesterone treatment. The maximum offset between the predicted curve and the ideal curve was 0.083 , and the minimum offset between the model and the ideal model was 0.021 . The height of the linear curve was close to that of the ideal curve. The $U$ test showed $\mathrm{P}=0.898$, greater than 0.05 , indicating that the nomogram model passed the calibration test. The receiver operating characteristic curve was 0.886 .

Conclusions: Our nomogram based on age, BMI, polyp size, progesterone treatment, and endometrial thickening accurately predicted the risk of polyp recurrence after endometrial polypectomy and can be applied in clinical practice.

Keywords: Calibration curves; endometrial polyps; nomograms; recurrence

Submitted Aug 31, 2021. Accepted for publication Oct 29, 2021.

doi: 10.21037/apm-21-2747

View this article at: https://dx.doi.org/10.21037/apm-21-2747 


\section{Introduction}

Endometrial polyps are localized hyperplasia of endometrial glandular tissue, stromal tissue, and other tissues that expand into the uterine cavity (1). The prevalence of endometrial polyps is between $7.8 \%$ and $34.9 \%$ (2), and they vary in size from a few millimeters to a few centimeters, can be single or multiple, asymptomatic, or a cause of infertility, irregular bleeding, or malignancy $(3,4)$. Hysteroscopic endometrial polypectomy is the primary treatment $(5,6)$, but the risk of postoperative recurrence is high (7). Studies have pointed out that the more endometrial polyps and the longer the follow-up time, the higher the risk of polyps recurrence after surgery. The type of endometrial polyps has nothing to do with the possibility of polyps recurrence. The reason for the high recurrence rate of endometrial polyps after surgery is still unclear. It may be related to inflammation, high estrogen levels, inappropriate selection of surgical methods, endocrine disorders, obesity, and age. The main risk factors for postoperative recurrence of endometrial polyps include the incomplete removal of the polyp pedicle during the operation, the postoperative local high estrogen status, the age of the patient, and the size of the polyp. How to accurately evaluate the risk of postoperative recurrence is a clinical problem that needs to be solved urgently. The purpose of this study was to screen the risk factors of recurrence after endometrial polypectomy, establish a nomogram, predict the risk of polyp recurrence, and provide a reference basis for clinical decision-making. We present the following article in accordance with the TRIPOD reporting checklist (available at https://dx.doi. org/10.21037/apm-21-2747).

\section{Methods}

\section{Case enrollment}

We enrolled 133 patients with endometrial polyps and endometrial polypectomy under hysteroscopy between January 2012 to June 2020, using the following inclusion criteria: (I) age between 18 and 65 years; (II) first diagnosis of endometrial polyps; (III) underwent hysteroscopic cold knife resection of endometrial polyps and diagnostic curettage; (IV) voluntary participation in the study and signed informed consent. The exclusion criteria were: (I) malignant tumors; (II) concurrent cardiovascular and cerebrovascular diseases; (III) inability to communicate normally; (IV) diagnosed with other uterine diseases. All procedures performed in this study involving human participants were in accordance with the Declaration of Helsinki (as revised in 2013). The study was approved by the Ethics Committee of The International Peace Maternity and Child Health Hospital, School of Medicine, Shanghai Jiao Tong University (No. GKLW 2019-08) and informed consent was obtained from all the patients.

\section{Surgical methods}

Hysteroscopic cold knife resection of endometrial polyps and diagnostic curettage was performed under general anesthesia; $0.9 \%$ normal saline was used for uterine distention with a pressure of $100 \mathrm{mmHg}$. The basal part of the polyp was completely removed to include the basal layer. The removed tissues were sent for pathological examination and confirmation of endometrial polyp.

\section{Follow-up}

Postoperatively, patients underwent B-ultrasound examination every 3 months. If suspicious endometrial polyps were detected, the patient had repeat surgery and if the pathological diagnosis was endometrial polyp, it was defined as recurrence. All patients were followed up for more than 12 months, and the average follow-up time was $46.37 \pm 5.14$ months.

\section{Statistical analysis}

The patients' clinical data were recorded, including age at first operation, body mass index (BMI), reproductive history, menopause, abnormal uterine bleeding, and timing of operation (first half or second half of menstruation cycle). Preoperative drug treatment, polyp size, endometrial thickening, and the pathology results (endometrial polyps and polypoid growth) were also recorded. During the follow-up period, whether postoperative progesterone treatment was administered and whether there was recurrence of polyps were recorded.

The data were statistically analyzed by R software (v3.5.1) and related $\mathrm{R}$ package. Count data are expressed as cases (percentage), and the chi-square test was used. Measurement data are expressed as mean and standard deviation, and an independent sample $t$-test was used. Logistic regression analysis was used to screen independent risk factors. Using machine learning, based on logistic regression analysis, the variables are transformed to make the variable and the prediction target form a linear relationship, and a 


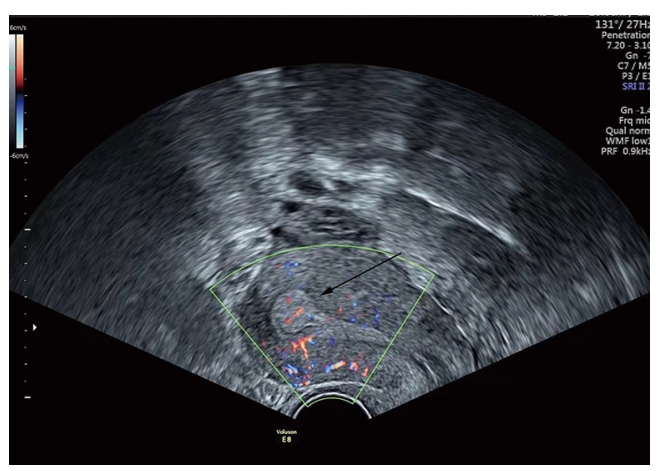

Figure 1 B-ultrasound image of recurrence after endometrial polypectomy. The black arrow marks the location of the polyp.

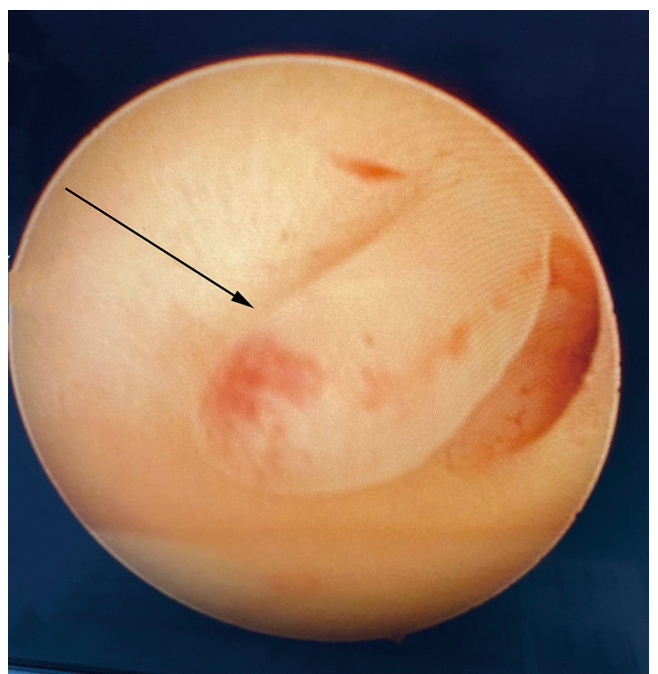

Figure 2 Hysteroscopic image of recurrence after endometrial polypectomy. The black arrow marks the location of the polyp.

nomogram for predicting the probability of adverse events is constructed based on this. The nomogram was verified by a fitting curve.

\section{Results}

\section{General data}

A total of $9(6.77 \%)$ cases were lost to follow-up, so $124(93.23 \%)$ cases were finally included in this study, comprising $98(79.03 \%)$ cases of recurrence after endometrial polypectomy, and $26(20.97 \%)$ cases of nonrecurrence. Of these, $82(66.13 \%)$ were aged $\geq 35$ years at the first operation, and $42(33.87 \%)$ were aged $<35$ years at the first operation; 18 (14.52\%) had given birth $\geq 2$ times and $106(85.48 \%)$ had $<2$ times. There were $23(18.55 \%)$ cases of $\geq 3$ abortions, and $101(81.45 \%)$ cases of $<3$. With regard to body weight, 54 (43.55\%) had BMI $\geq 28 \mathrm{~kg} / \mathrm{m}^{2}$, and 70 $(56.45 \%)$ were $<28 \mathrm{~kg} / \mathrm{m}^{2}$. There were $20(16.13 \%)$ cases of polyps $\geq 2 \mathrm{~cm}, 20$ (16.13\%) cases of menopause, 39 (31.45\%) cases of abnormal uterine bleeding, and 17 (13.71\%) cases of preoperative drug treatment at the first operation; $37(29.84 \%)$ patients were treated with progesterone postoperative at the first operation, and $68(54.84 \%)$ patients had endometrial thickening. The B-ultrasound image of recurrence after endometrial polypectomy was shown in Figure 1. The hysteroscopic image of recurrence after endometrial polypectomy was shown in Figure 2.

\section{Comparison of clinical factors in recurrence and non- recurrence cases}

In the group with recurrence after endometrial polypectomy, the proportion of patients aged $<35$ years was lower than in the non-recurrence group $(\mathrm{P}=0.004)$, the proportion of patients with $\mathrm{BMI}<28 \mathrm{~kg} / \mathrm{m}^{2}$ was lower than in the non-recurrence group $(\mathrm{P}=0.028)$, the proportion of patients with polyps $<2 \mathrm{~cm}$ in the recurrence group was lower than the non-recurrence group $(\mathrm{P}=0.027)$, and the ratio of patients treated with progesterone in the recurrence group was lower than in the non-recurrence group $(\mathrm{P}=0.003)$. In contrast, the proportion of patients with endometrial thickening was higher in the recurrence group than in the non-recurrence group $(\mathrm{P}=0.006)$ (Table 1).

\section{Independent risk factor screening}

In this study, recurrence after endometrial polypectomy was taken as the dependent variable (no recurrence $=0$, recurrence $=1)$, while age $(<35$ years $=0, \geq 35$ years $=1)$, BMI $\left(<28 \mathrm{~kg} / \mathrm{m}^{2}=0, \geq 28 \mathrm{~kg} / \mathrm{m}^{2}=1\right)$, polyp size $(<2 \mathrm{~cm}=0$, $\geq 2 \mathrm{~cm} \mathrm{=1}$ ), postoperative progesterone treatment (not used $=0$, used $=1$ ) after first hysteroscopy, and endometrial thickening (no thickening $=0$, thickening $=1$ ) were independent variables, and logistic analysis was performed. The results showed that age, BMI, polyp size and endometrial thickening were independent risk factors for recurrence after endometrial polypectomy [odds ratio (OR) $>1, \mathrm{P}<0.05]$. Progesterone therapy after first hysteroscopy was an independent protective factor for recurrence after endometrial polypectomy $(\mathrm{OR}<1, \mathrm{P}<0.05)$ (Table 2). 
Table 1 Comparison of clinical factors in cases of recurrence and non-recurrence of endometrial polyps

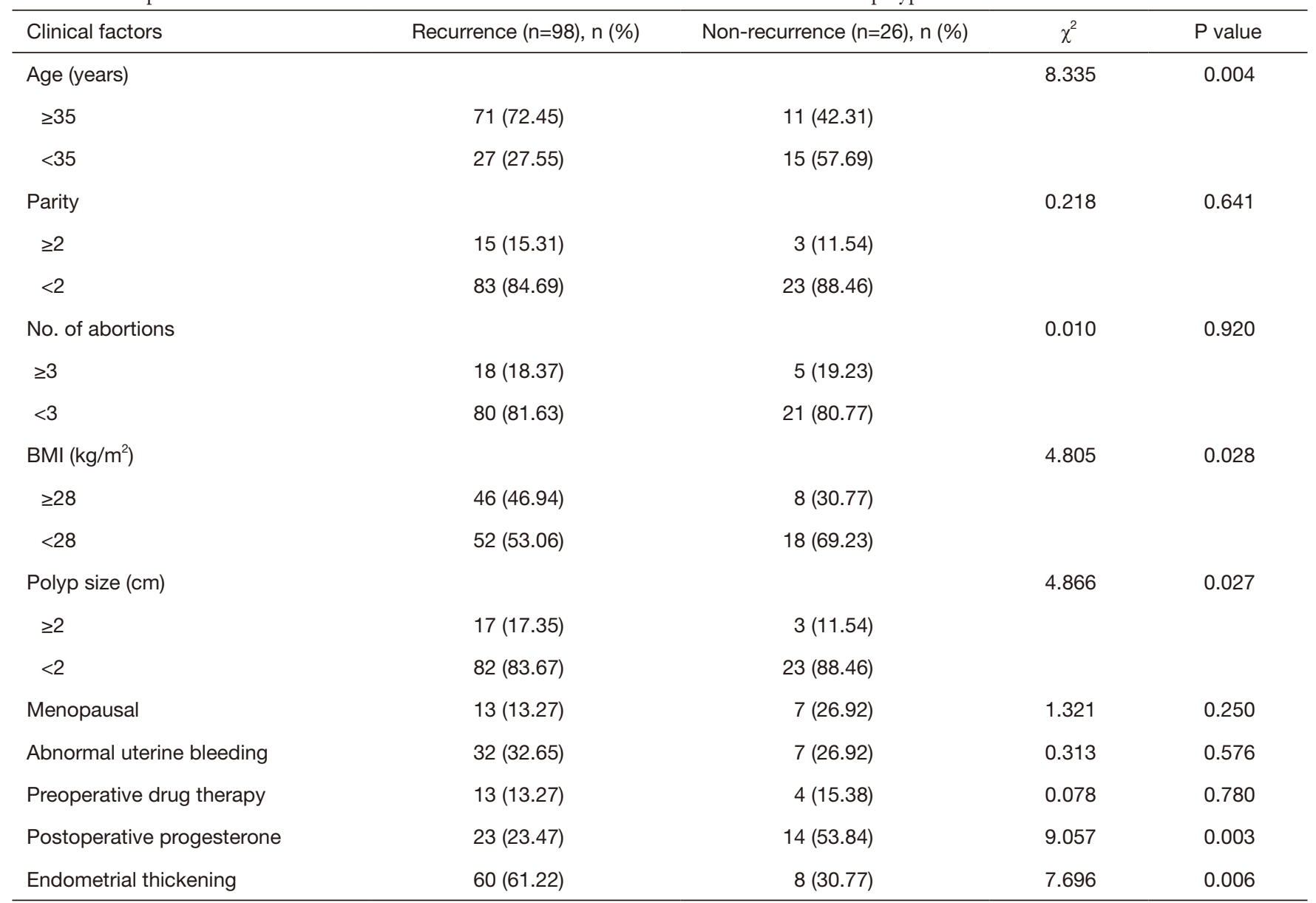

BMI, body mass index.

Table 2 Logistic analysis of risk factors for recurrence after endometrial polypectomy

\begin{tabular}{lcc}
\hline Variable & OR $(95 \% \mathrm{Cl})$ & P value \\
\hline Age & $1.32(1.08,1.49)$ & 0.003 \\
BMI & $1.17(0.86,1.31)$ & 0.024 \\
Polyp size & $1.14(0.93,1.63)$ & 0.031 \\
Postoperative progesterone & $0.64(0.55,1.02)$ & 0.004 \\
Endometrial thickening & $1.29(0.96,1.37)$ & 0.004 \\
\hline
\end{tabular}

$\mathrm{BMI}$, body mass index; OR, odds ratio; $\mathrm{Cl}$, confidence interval.

\section{Construction of nomogram for recurrence risk after endometrial polypectomy}

We used logistic regression analysis to establish the nomogram of recurrence risk assessment after endometrial polypectomy, using the variables of age, BMI, polyp size, postoperative use of progesterone therapy, and endometrial thickening (Figure 3).

\section{Nomogram calibration curve evaluation}

The calibration curves of the nomogram prediction models were compared. The maximum offset between the nomogram and ideal curves constructed in this study was 0.083 , and the minimum offset between the model and the ideal model was 0.021 . The height of the nomogram curve was close to that of the ideal curve. The unreliability (U) test showed $\mathrm{P}=0.898$, greater than 0.05 , thus the nomogram model passed the calibration test. The area under the receiver operating characteristic curve (ROC) was 0.886 , indicating that the model's predictive ability was good (Figure 4). 


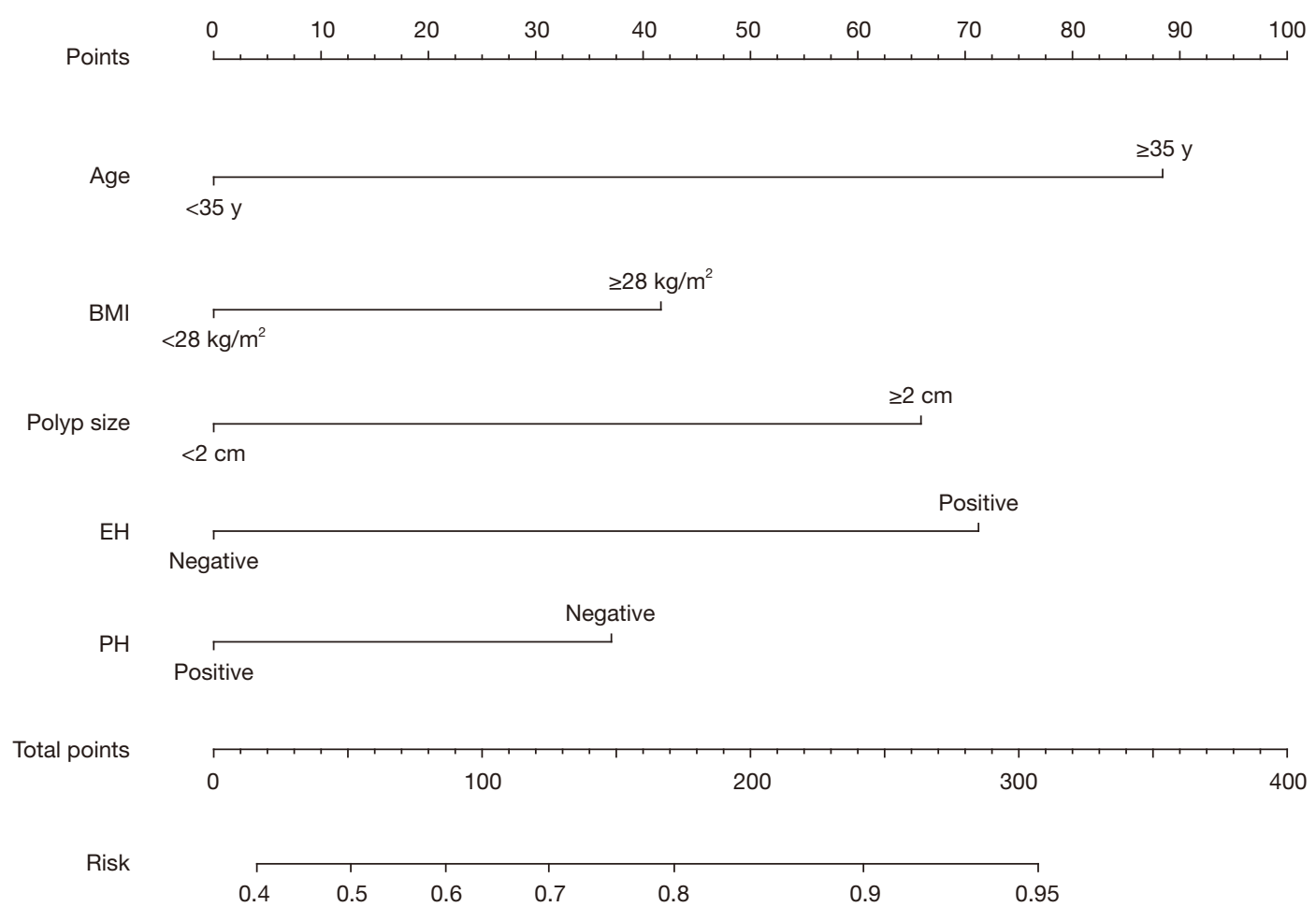

Figure 3 Nomogram of recurrence risk after endometrial polypectomy. BMI, body mass index; EH, endometrial hyperplasia; PH, progestational hormone.

\section{Discussion}

Endometrial polyps are localized endometrial hyperplasia composed of endometrial stroma, glands, and blood vessels, with a prevalence ranging from $7.8 \%$ to $34.9 \%$; the incidence of malignant transformation of endometrial polyps ranges from $0 \%$ to $12.9 \%$ (2). Hysteroscopic endometrial polypectomy is a relatively safe and simple operation that can effectively remove polyps (8-10), but studies have shown that the recurrence rate of endometrial polyps is as high as $46 \%$, so it is crucial to identify the related risk factors for effective monitoring and timely clinical intervention $(7,11)$. Factors that may be associated with recurrence have been analyzed in other clinical studies. Yang et al. evaluated 168 patients with premenopausal endometrial polyps who underwent hysteroscopic polypectomy and found that both polyp diversity and duration of follow-up significantly correlated with recurrence (11). Preutthipan et al. found that the recurrence rate of polyps was directly related to hysteroscopic surgery (12), but this conclusion is controversial. Others have shown that different surgical methods under hysteroscopy had no significant effect on the postoperative recurrence rate of endometrial polyps (13). Zhu et al. (14) observed that patients with recurrent and non-recurrent polyps showed a difference in the expression of interferon- $\gamma$, interleukin-17, and Foxp3. By evaluating the cellular immune system's role, they considered that a $T$ cell imbalance was related to the occurrence of recurrent polyps. All these findings suggest that the cause of endometrial polyp recurrence may be multifactorial, and further research is needed to clarify its pathogenesis.

Compared with previous studies, we included more clinical variables, including those affecting the recurrence rate of endometrial polyps. We included BMI as a variable rather than weight because other studies have confirmed that the risk factors for the development of endometrial polyps include age, tamoxifen use, obesity, and hypertension $(14,15)$. BMI can better assess whether a patient is obese than body weight. Polyp size is related to polyp proliferation, and the proliferation rate of endometrial polyps $>15 \mathrm{~mm}$ is $14.8 \%$, and while that of endometrial polyps $<15 \mathrm{~mm}$ is $7.7 \%$ (16). Therefore, we included polyp size as a variable of polyp recurrence. We found significant differences in age, BMI, polyp size, progesterone treatment, 


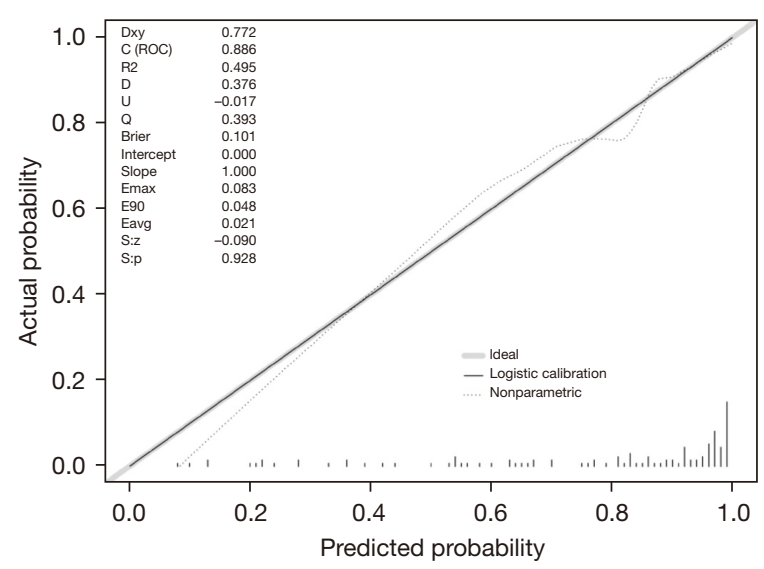

Figure 4 Nomograph calibration curve. The dotted line represents the nomograph model, and the solid line represents the ideal model. The abscissa represents the predicted risk, and the ordinate represents the actual risk. Dxy, variance of a random variable; $\mathrm{C}$ (ROC), area under curve (ROC); R2, r-square test; D, D-normality test; U, unreliability test; Q, q test; Brier, Brier score; Intercept, intercept function; Slope, slope function; Emax, maximum deviation between prognosis evaluation model and ideal model; E90, deviation prognosis evaluation model and ideal model (predicted probability =0.9); Eavg, minimum offset between the model and the ideal model; S:z, z-value; S:p, P value; ROC, receiver operating characteristic curve.

and endometrial thickening between the recurrent and nonrecurrent groups after endometrial polypectomy. We found that age, BMI, polyp size, and endometrial thickening were independent risk factors for recurrence after endometrial polypectomy through logistic regression analysis. Postoperative progesterone therapy was an independent protective factor for recurrence after endometrial polypectomy in this study, which was consistent with some previous research results. Paradisi et al. found that recurrence after endometrial hysteroscopic polypectomy was related to non-atypical concurrent hyperplasia and had nothing to do with appearance, age, parity, weight, or other demographic characteristics (17). The use of progesterone after endometrial polypectomy could significantly reduce the recurrence rate of polyps, which has been confirmed in some previous studies $(18,19)$. After identifying the independent risk factors of endometrial polyp recurrence, we constructed a nomogram for predicting polyp recurrence using logistic regression analysis and verified the calibration curve. The nomogram curve was highly fitted with the calibration ideal curve, the maximum offset value was 0.083 , and the area under the ROC curve was 0.886 . These results confirmed the reliability and accuracy of the prediction results of the nomogram.

Surgical resection is the main treatment method for endometrial polyps. At present, hysteroscopic endometrial polypectomy is the preferred treatment method. Its advantages include the ability to remove polyps under direct vision and reduce the risk of intraoperative surgery. In addition, we also recommend using a cold knife for resection. The rate of endometrial recurrence, menstrual volume, recurrence time, and endometrial thickness during ovulation after cold knife resection were better than those of diagnostic curettage. Although there are no clinical guidelines to follow, the results of clinical studies in recent years suggest that for patients with endometrial polyps, hysteroscopic resection combined with drug adjuvant therapy can be used. The current postoperative adjuvant treatment methods include progesterone, compound oral contraceptives, anti-progesterone drugs, levonorgestrel intrauterine sustained-release system and so on.

In conclusion, the nomogram constructed in this study based on age, BMI, polyp size, whether postoperative progesterone treatment is used, and whether the endometrium is thickened can accurately predict the risk of polyp recurrence after endometrial polypectomy and is practical for use in the clinic.

\section{Acknowledgments}

Funding: The research was supported by MedicalEngineering Cross Fund from Shanghai Jiao Tong University (No. ZH2018QNB17).

\section{Footnote}

Reporting Checklist: The authors have completed the TRIPOD reporting checklist. Available at https://dx.doi. org/10.21037/apm-21-2747

Data Sharing Statement: Available at https://dx.doi. org/10.21037/apm-21-2747

Conflicts of Interest: All authors have completed the ICMJE uniform disclosure form (available at https://dx.doi. org/10.21037/apm-21-2747). The authors have no conflicts of interest to declare.

Ethical Statement: The authors are accountable for all aspects of the work in ensuring that questions related 
to the accuracy or integrity of any part of the work are appropriately investigated and resolved. All procedures performed in this study involving human participants were in accordance with the Declaration of Helsinki (as revised in 2013). The study was approved by the Ethics Committee of The International Peace Maternity and Child Health Hospital, School of Medicine, Shanghai Jiao Tong University (No. GKLW 2019-08) and informed consent was obtained from all the patients.

Open Access Statement: This is an Open Access article distributed in accordance with the Creative Commons Attribution-NonCommercial-NoDerivs 4.0 International License (CC BY-NC-ND 4.0), which permits the noncommercial replication and distribution of the article with the strict proviso that no changes or edits are made and the original work is properly cited (including links to both the formal publication through the relevant DOI and the license). See: https://creativecommons.org/licenses/by-nc-nd/4.0/.

\section{References}

1. Nijkang NP, Anderson L, Markham R, et al. Endometrial polyps: Pathogenesis, sequelae and treatment. SAGE Open Med 2019;7:2050312119848247.

2. Ciscato A, Zare SY, Fadare O. The significance of recurrence in endometrial polyps: a clinicopathologic analysis. Hum Pathol 2020;100:38-44.

3. Clark TJ, Stevenson H. Endometrial Polyps and Abnormal Uterine Bleeding (AUB-P): What is the relationship, how are they diagnosed and how are they treated? Best Pract Res Clin Obstet Gynaecol 2017;40:89-104.

4. Wong M, Crnobrnja B, Liberale V, et al. The natural history of endometrial polyps. Hum Reprod 2017;32:3405 .

5. Salim S, Won H, Nesbitt-Hawes E, et al. Diagnosis and management of endometrial polyps: a critical review of the literature. J Minim Invasive Gynecol 2011;18:569-81.

6. de Azevedo JM, de Azevedo LM, Freitas F, et al. Endometrial polyps: when to resect? Arch Gynecol Obstet 2016;293:639-43.

7. Pavone ME. Predicting the recurrence of endometrial polyps: a commentary. Fertil Steril 2018;109:445.

8. Soljačić Vraneš H, Djaković I, Vrljičak M, et al. Histopathologic findings in women undergoing hysteroscopic resection of endometrial polyps and uterine myomas. Acta Clin Croat 2019;58:627-31.

9. Kuzel D, Lisa Z, Fanta M, et al. Hysteroscopic management of endometrial polyps and submucous leiomyomas in women using a levonorgestrel-releasing intrauterine system. Minim Invasive Ther Allied Technol 2021. [Epub ahead of print].

10. Su L, You L, Huang HP. Risk Factors of Intrauterine Adhesion after Hysteroscopic Resection of Endometrial Polyps. Zhongguo Yi Xue Ke Xue Yuan Xue Bao 2017;39:812-6.

11. Yang JH, Chen CD, Chen SU, et al. Factors Influencing the Recurrence Potential of Benign Endometrial Polyps after Hysteroscopic Polypectomy. PLoS One 2015;10:e0144857.

12. Preutthipan S, Herabutya Y. Hysteroscopic polypectomy in 240 premenopausal and postmenopausal women. Fertil Steril 2005;83:705-9.

13. Ceci O, Franchini M, Cardinale S, et al. Comparison of endometrial polyp recurrence in fertile women after office hysteroscopic endometrial polypectomy using two widely spread techniques. J Obstet Gynaecol Res 2020;46:2084-91.

14. Zhu Y, Du M, Yi L, et al. CD4+ T cell imbalance is associated with recurrent endometrial polyps. Clin Exp Pharmacol Physiol 2018;45:507-13.

15. Serhat E, Cogendez E, Selcuk S, et al. Is there a relationship between endometrial polyps and obesity, diabetes mellitus, hypertension? Arch Gynecol Obstet 2014;290:937-41.

16. Lasmar BP, Lasmar RB. Endometrial polyp size and polyp hyperplasia. Int J Gynaecol Obstet 2013;123:236-9.

17. Paradisi R, Rossi S, Scifo MC, et al. Recurrence of endometrial polyps. Gynecol Obstet Invest 2014;78:26-32.

18. Wang X, Huang XH. Progress on surgical treatment and prevention of recurrence in endometrial polyps. Zhonghua Fu Chan Ke Za Zhi 2011;46:307-10.

19. Li F, Wei S, Yang S, et al. Post hysteroscopic progesterone hormone therapy in the treatment of endometrial polyps. Pak J Med Sci 2018;34:1267-71.

(English Language Editor: K. Brown)

Cite this article as: Liu J, Liang Y, Ouyang J, Yang S. Analysis of risk factors and model establishment of recurrence after endometrial polypectomy. Ann Palliat Med 2021;10(11):1162811634. doi: 10.21037/apm-21-2747 